\title{
Mapa del Proceso de Internacionalización de las Instituciones de Educación Superior Colombianas
}

\author{
Moises Briñez ${ }^{1}$ \\ https://orcid.org/0000-0002-1126-017X \\ Sebastián Gómez ${ }^{2}$ \\ Juan Guerra ${ }^{3}$ \\ Yerferson Betancurt ${ }^{4}$ \\ Juan Camilo Isaza ${ }^{5}$
}

\section{RESUMEN}

Recebido: 17.10.2019

Aceito: 04.12.2019

Publicado: 13.01 .2020

El objetivo de la presente investigación estuvo orientado a estructurar el mapa del proceso de internacionalización de las Instituciones de Educación Superior Colombianas (IESC), de tal forma, se identificaron las actividades estratégicas, clave y de apoyo, que realmente generan valor. Los principales autores considerados son: Sorolla (2019), Mayer y Catalano (2019), Castro y Wolf (2018), Ganga, Pérez y Mansilla (2018), Hoyos y Villamizar (2018), Colciencias (2017), Colotta, Lavallen y Presa (2017), Pérez (2009), entre otros. La investigación se considera de tipo descriptiva, bajo un enfoque cualitativo, con un diseño no experimental, transeccional. En una primera fase se procedió a revisar modelos de internacionalización de IES en países como China, Argentina, Costa Rica, México, Cuba, entre otros. Seguidamente, se desarrollaron mesas de trabajo para clasificar las actividades que componen el mapa de procesos. A partir de este estudio, se realizaron una serie de entrevistas abiertas al personal del área de internacionalización de las principales IES de ubicadas en el Departamento de Antioquia. Finalmente, se propone la estructura del proceso de Internacionalización, indicando no solo las actividades fundamentales, sino también la interrelación estratégica que las mismas deben mantener, para asegurar la efectividad del mismo.

Palabras clave: mapa de procesos, internacionalización, actividades estratégicas, clave y de apoyo, interrelación estratégica.

\section{Mapa do Processo de Internacionalização das Instituições colombianas de Ensino Superior \\ RESUMO}

O objetivo da presente investigação esteve orientado a estruturar o mapa do processo de internacionalização das Instituciones de Educación Superior Colombianas (IESC), de tal forma, se identificaram as actividades estratégicas, chave e de apoyo, que realmente agregam valor. Os principais autores considerados são: Sorolla (2019), Mayer e Catalano (2019), Castro e Wolf (2018), Ganga, Pérez e Mansilla (2018), Hoyos e Villamizar (2018), Colciencias (2017), Colotta, Lavallen e Presa (2017), Pérez (2009), entre outros. A investigação considera-se de tipo descriptiva, uma abordagem qualitativa, com um desenho não experimental. Numa primera fase se procedeu a revisar os modelos de internacionalização de IES em países como: China, Argentina, Costa Rica, México, Cuba, entre outros. Seguidamente, desenvolveram-se actividades laboráis para classificar as actividades que compõem o mapa de processos. A partir deste estudo, realizaram-se una serie de entrevistas abertas ao pessoal da área de internacionalização das principais IES de localizadas no Departamento de Antioquia. Finalmente, propõem-se a estructura do processo de Internacionalização, indicando não só as actividades fundamentais, mas também a inter-relação estratégica que as mesmas devem manter, para assegurar a efectividade do mesmo.

Palavras-chave: Mapa de processos, Internacionalização, Actividades estratégicas, chave e de apoio, inter-relação estratégica.

\footnotetext{
${ }^{1}$ Universidad EIA, Colombia. Correo electrónico: moises.brinez@eia.edu.co

${ }^{2}$ Universidad EIA, Colombia

${ }^{3}$ Universidad EIA, Colombia

${ }^{4}$ Universidad EIA, Colombia

${ }^{5}$ Universidad EIA, Colombia
} 


\section{Map of the Internationalization Process of Colombian Higher Education Institutions}

\section{ABSTRACT}

The objective of the present investigation was oriented to structure the map of the internationalization process of the Colombian Higher Education Institutions (IESC), in this way, the strategic, key and support activities that really generate value were identified. The main authors considered are: Sorolla (2019), Mayer and Catalano (2019), Castro and Wolf (2018), Ganga, Pérez and Mansilla (2018), Hoyos and Villamizar (2018), Colciencias (2017), Colotta, Lavallen and Presa (2017), Pérez (2009), among others. Research is considered descriptive, under a qualitative approach, with a non-experimental, transectional design. In a first phase, IES internationalization models were reviewed in countries such as China, Argentina, Costa Rica, Mexico, Cuba, among others. Next, work tables were developed to classify the activities that make up the process map. From this study, a series of open interviews were conducted with the staff of the internationalization area of the main IES located in the Department of Antioquia. Finally, the structure of the Internationalization process is proposed, indicating not only the fundamental activities, but also the strategic interrelation that they must maintain, to ensure its effectiveness.

Keywords: process map, internationalization, strategic, key and support activities, strategic interrelation.

\section{Introducción}

Actualmente, la realidad empresarial es caracterizada por altos niveles de complejidad, dinamismo, pero, sobre todo, competitividad. Las tendencias han cambiado significativamente; por tal razón, para sobrevivir en este contexto, ya no es suficiente con cumplir con calidad y expectativas; es necesario exceder las exigencias del cliente consiguiendo resultados únicos, y el ámbito académico universitario no es la excepción. La realidad empresarial, se caracteriza por un marcado nivel de globalización, generando una nueva concepción en la manera formar profesionales competitivos, la cual implica la superación de barreras nacionales como requisito para establecer diferencias sostenibles.

Bajo este contexto, el proceso de internacionalización de las Instituciones de Educación Superior (IES), implica dimensionar la función educativa hacia una visión global, intercultural, capaz de combinar esfuerzos desde la academia, para garantizar la formación de una generación con una mirada holística, humanista, solidaria y responsable ante la sociedad.

A nivel continental, en América Latina, las cifras reales del impacto o efectos generados por este proceso, son escasas, por no decir que en muchos países son totalmente ausentes; eso lleva a que las políticas diseñadas para promover el avance de la actividad internacional, muchas veces sean en vano, por no estar articulados de forma armoniosa entre gobierno, academia y sociedad, disminuyendo la posibilidad de aportar un valor agregado en el ejercicio de la formación académica. Esto último hace inferir, que no existe un adecuado proceso de internacionalización, capaz de coordinar cada una de las actividades en las cuales se involucran las instituciones de educación superior colombianas. Por ello, este ámbito académico no escapa de la necesidad de mejorar y optimizar sus procesos a fin de identificar las ineficacias, posibilidades de mejora, o bajos beneficios que generan los mismos.

Ahora bien, para entender dichos procesos es necesario visualizar y apreciar las interrelaciones entre ellos, al igual que los subprocesos, así como las actividades que contribuyen a perfeccionar el resultado que la institución desea alcanzar, de esta manera, cualquier posibilidad de mejora puede ser evidente de forma clara y propiciar el inicio de acciones correspondientes.

En este sentido, surge la gestión por procesos, la cual busca generalizar la revisión de los procesos como una práctica sistemática, además, como menciona Benavides y Quintana (2003), sirve como un esquema que permite organizar los esfuerzos y la utilización de los recursos - sean tangibles o intangibles - para lograr la satisfacción balanceada de todos los entes vinculados a cada uno de los procesos que definen al sistema organizacional.

Son tales los beneficios del esquema que supone la gestión por procesos que la norma internacional ISO 9001, lo considera como un requisito del sistema organizacional, señalando que "se promueve la adopción de un enfoque a procesos para el desarrollo, implantación y mejora de la eficacia y eficiencia en un sistema de gestión de calidad” (Bello, 2006: 59). 
Apoyando lo anterior, Pérez (2009) indica que la norma ISO 9001 requiere que se establezca la interacción entre los procesos, siendo una de las mejores formas de solucionarlo la elaboración del mapa de proceso, actuando como una herramienta que permite representar los procesos relevantes para satisfacer al cliente y lograr los objetivos de la empresa al desagregar su cadena de valor en actividades estratégicas, clave y de apoyo, cada una de las cuales se descompone en un conjunto de procesos interconectados entre sí.

A partir de lo anterior, esta investigación propone el mapa del proceso de internacionalización de las IES Colombianas, de tal forma, se identificaron las actividades estratégicas, clave y de apoyo, que realmente generan valor; constituyendo un avance para desarrollar en una futura investigación, un modelo de gestión capaz de monitorear y medir la efectividad de cada actividad.

Para el desarrollo de este trabajo, las técnicas de recolección de datos estuvieron conformadas por entrevistas, para la obtención de información práctica que se utilizó para conocer la variable mapa del proceso de internacionalización, aplicado a algunas instituciones de educación superior, además se hizo uso de la observación documental, fundada en la revisión de libros, folletos, documentos, revistas y conferencias que dieron todo el soporte necesario para culminar el trabajo.

\section{El proceso de Internacionalización en las Instituciones de Educación Superior Colombianas (IES).}

Para empezar a hablar del proceso de internacionalización de Colombia primero tenemos que conocer un poco de su estructura organizacional en el ámbito de la educación superior. La educación superior en Colombia tiene los siguientes niveles: Técnico profesional, tecnólogo y profesional además de los niveles de especialización que se dividen en especializaciones, maestrías y doctorados.

Se sabe que en total el 29\% de las IES son universidades, el $42 \%$ instituciones universitarias, el $18 \%$ instituciones tecnológicas y $12 \%$ instituciones técnicas, lo cual indica que la tendencia de la educación superior en Colombia es a realizar estudios universitarios, sobre los técnicos o los tecnólogos. Además, se puede afirmar que la gran mayoría de las IES son instituciones privadas sin ánimo de lucros las cuales ofrecen 11.203 programas académicos (Castro y Wolff, 2018).

Paralelamente, se muestra que el panorama en lo referente a la movilidad internacional no es tan prometedor, ya que únicamente el 0,5\% de los estudiantes inscritos en las IES realizan este trámite. Sin embargo, producto de todos los esfuerzos realizados por las diversas entidades públicas, esa movilidad académica -entre 2011 y 2015- creció en promedio un 31\% anual.

Por tal motivo, el tema de la internacionalización es considerado por el gobierno como un tema central de desarrollo y proyección; por lo tanto, se busca generar programas para aumentar el número de estudiantes que quieran realizar estudios en el exterior, y no solo de los estudiantes sino también del personal docente para que se capaciten y adquieran nuevos conocimientos en el ámbito de las prácticas educativas y la investigación.

Como se mencionó anteriormente el gobierno y algunas instituciones públicas están empleando sus esfuerzos para que el proceso de internacionalización tenga mayor alcance, a continuación, se mostrará algunas de ellas según la opinión de Castro y Wolff (2018):

Ministerio de educación: El ministerio de educación desde el año 2009 ha empleado diversas estrategias para que el territorio colombiano tenga más presencia a nivel internacional y sea un destino más atractivo para el intercambio cultural y de conocimiento, para esto se tuvieron tres focos principales que son:

- Mostrar a Colombia como destino académico de calidad: Esto es importante ya que permite la visualización de Colombia a nivel internacional, también ayuda a generar convenios con diversos países para facilitar la movilización académica tanto de estudiantes como de docentes e investigadores. Para llevar a cabo esta iniciativa se realizó participación en ferias internacionales que se utilizaban como espacios para promocionar el país. Además, se 
realizaron visitas a instituciones de educación superior y gobiernos de otros países con el mismo fin.

- Construcción y fortalecimiento de capacidades en internacionalización: Esto ayuda a que el personal académico de las IES en Colombia, tengan un mayor conocimiento acerca de los procesos de internacionalización y adquieran la capacidad de dar información al resto de personas para hacerlo más atractivo. Para esto se generaron desde el año 2009 programas de acompañamiento para el fortalecimiento de capacidades de internacionalización, brindando asesorías a IES acerca de todo lo referente al tema.

- Alianzas internacionales: Como se mencionó anteriormente estas alianzas facilitan el proceso de internacionalización ya que genera puntos de contacto para la realización del proceso.

ICETEX: éste enfocó sus esfuerzos en lo que se refiere a la movilidad académica y la gestión de la internacionalización del currículo, las cuales se consideran como dos de las actividades claves del proceso de internacionalización en Colombia, para llevar a cabo estas iniciativas se enfocaron en la cooperación con IES locales y en el exterior, para permitir el acceso a becas a estudiantes colombianos. Esto resultó muy importante ya que permite que estudiantes que no posean los recursos suficientes para realizar un proceso de internacionalización por su cuenta, tengan la posibilidad de ser partícipes de este proyecto. De estas becas se beneficiaron 4775 estudiantes, los cuales se repartieron en las siguientes proporciones: 23\% becas en el pacífico, y más de 41\% en desarrollo de programas de maestría (Castro y Wolff, 2018).

Por otra parte, para llevar a cabo la gestión de la internacionalización del currículo se desarrollaron dos programas. El primero se conoció como asistentes de idiomas que dentro de sus planes considera la movilidad académica de docentes de idiomas extranjeros para incentivar y contribuir el fortalecimiento de los departamentos de idiomas de los colegios y IES; lo cual permite que el idioma deje de ser una barrera para llevar a cabo la internacionalización. Por medio de este programa se movilizaron más de 700 asistentes desde el exterior (Hoyos y Villamizar, 2018).

El segundo programa se conoció como profesores invitados (Fellows Colombia), cuyo principal enfoque es la movilidad de expertos a IES y centros de investigación para fortalecer programas de investigación, contribuyendo a ahondar más en temas referentes a los avances tecnológico y de investigación en las IES. A diferencia del primer programa este está más enfocado a sectores más avanzados de la educación y contó con más de 600 expertos en el área de investigación de los cuales el 30\% se movilizaron en el año 2016 (Hoyos y Villamizar, 2018).

Departamento de ciencias, tecnología e innovación: El departamento de ciencias, tecnología e innovación también conocido como Colciencias, se enfoca en promover la internacionalización de la investigación y la innovación, a través del fortalecimiento de las relaciones con socios internacionales para el desarrollo de ciencia tecnología e innovación, para esto se emplearon diversas estrategias como lo son: la cooperación internacional, que permitió el trabajo conjunto de universidades locales y extranjeras para el desarrollo de nuevas prácticas innovadoras y otorgar visualización a Colombia en el extranjero. Cabe aclarar que estas alianzas no se gestionan exclusivamente desde Colombia sino también desde diversos territorios como lo son Norteamérica, Asia, África, Oceanía e Iberoamérica (Colciencias, 2017).

También se fomentó la participación de instituciones colombianas en programas de la comunidad europea cuyo fin fue adquirir capacitaciones para las IES colombianas por parte de instituciones en el extranjero. Gracias a esto se generaron once puntos de contacto a nivel nacional que son temáticos en áreas específicas de investigación. Aparte de las dos practicas anteriormente mencionadas se desarrollaron programas de modalidades de movilidad y pasantías de investigación, todo con el fin de mejorar y hacer más viable el proceso de internacionalización. Red Colombia Challenge Your knowledge: Esta red se encargó de promover la visibilidad nacional e internacional de las IES para adquirir la acreditación de alta calidad a nivel nacional, el objetivo era promover y diseminar la investigación y los programas académicos colombianos además de 
fortalecer la internacionalización de sus miembros y promover los procesos de calidad en las IES (Botero y Bolívar, 2015).

Plataforma de movilidad estudiantil y académica de alianza con el pacifico: ésta gestionó becas para todos los niveles de educación en toda Latinoamérica, es decir, que países como Colombia, Perú, chile y México son beneficiarios de este programa. Actualmente se otorgan cien becas anuales por país en una proporción de 75\% para pregrado y 25\% para postgrado (Castro y Wolff, 2018).

Programa Colombia cientifica: este programa comprende dos componentes, uno conocido como pasaporte para la ciencia, el cual otorga becas para programas de maestría y doctorado en universidades que ocupan los primeros puestos en el Academic Ranking Of World Universities. El segundo componente se conoce como ecosistema científico, el cual promueve el desarrollo de capacidades y redes de conocimiento en investigación docencia e innovación, además de la financiación de alianzas de universidades locales y extranjeras. (Instituto Colombiano de Crédito Educativo y Estudios en el Exterior Mariano Ospina Pérez, 2017).

Es oportuno mencionar lo que señalan Castro y Wolff (2018) que, en busca de esos resultados, en el año 2013 se crea una iniciativa por parte del consejo nacional de acreditación en el que proponen un factor de visibilidad nacional e internacional como requisito para que las instituciones superiores se acrediten como de alta calidad. A partir de lo anterior se puede aseverar que, en Colombia, el proceso de internacionalización de las IES se ha convertido en un asunto central en el desarrollo y proyección reciente del sistema educativo del país, no solo para las universidades sino también para el gobierno colombiano; dado que a través de la internacionalización se ha incrementado la calidad de la docencia, así como la investigación.

\section{Una aproximación a la Gestión por Procesos.}

Desde hace años, y hasta la actualidad existe una tendencia a estandarizar y mejorar los procesos de la manera más adecuada a las necesidades de la empresa, con el fin de lograr la satisfacción del cliente, a esta metodología se le conoce como gestión por procesos (BPM por sus siglas en ingles). Para comprender de una mejor manera, partimos de la definición del proceso administrativo. Este puede ser conceptualizado como un conjunto de pasos enfocados en reunir los pasos necesarios para ejecutar una actividad administrativa logrando los mejores resultados posibles.

Por otro lado, se puede conceptualizar como una serie de actividades secuenciales que lleva a cabo un productor, sobre un insumo, le agrega valor a este y genera un producto o servicio. En otras palabras, es un conjunto de actividades profundamente relacionadas que toman determinados insumos (entradas), los cuales son transformados en un resultado (salidas) o en un producto. Además, podría contener otras etapas o subprocesos.

Por definición, la gestión es un conjunto de operaciones que se efectúan para conducir y gestionar un negocio o una empresa. En conjunto, administrar un proceso es garantizar un resultado a través de los objetivos que, en términos de calidad se definen por medio de, costos, atención, disposición y seguridad.

Partiendo de lo anterior, la Gestión por procesos consiste en orientar la empresa hacia y desde el cliente, diseñando procesos efectivos que permitan satisfacer sus necesidades y expectativas (Harrington, 1993). Para Gómez (2009), el enfoque a procesos consiste en fragmentar el sistema en procesos, conocer y gestionar las relaciones existentes entre ellos con el fin de decidir y emprender mejoras individuales que sean necesarias.

Para lograr esta gestión se utiliza un método grafico conocido como mapa de procesos (MP), basado en identificar y clasificar los procesos de la empresa. Mapear un proceso implica listar y conocer primero todos los procesos de una organización y sus actividades, estudiando cómo se relacionan e interactúan entre si unos con otros.

El diseño de los mapas de procesos de una organización se observa durante la formulación de su plan estratégico corporativo, con el propósito de comprender mejor y más profundamente el 
trabajo y el desempeño de los procesos, así como las actividades en los que se halla involucrado, orientando una atención primordial a aquellos elementos clave de los mismos.

Los grafismos utilizados para hacer los mapas de procesos pasan determinados datos, por lo que, con apoyo de otras técnicas de gestión, vale la pena aprovecharlos como herramientas de comunicación; para lo cual han de ser fácilmente entendibles. Vale destacar que no existe un formato predeterminado para la construcción de un mapa de procesos, cada organización debe adoptar el diseño que mejor se adapte a la complejidad o sencillez de sus actividades.

El primer paso para poder generar uno es identificar los tipos de procesos existentes en la organización. En tal sentido Pérez (2009) define los tipos de procesos según su misión, los cuales se detallan a continuación:

- Procesos operativos: son aquellos cuya función básica está orientada en la combinación y transformación de insumos que permiten obtener el producto o suministrar el servicio, de acuerdo a las exigencias del cliente, ofreciendo un alto valor añadido. Por su naturaleza, son también los principales garantes de lograr los objetivos de la empresa.

- Procesos de apoyo: Facilitan las personas y los recursos físicos necesarios por el resto de procesos y acorde a los requisitos de sus clientes internos.

- Procesos de gestión: a través de actividades de evaluación, control, monitoreo y evaluación, garantizan el funcionamiento adecuado del resto del proceso, además provee la información que requiere para tomar decisiones y diseñar planes de mejora eficaces. Operan recolectando datos del resto de los procesos y transformándolos para convertirlos en información de valor para sus clientes internos, información clara, veraz, precisa, pertinente, y accesible .

- Procesos de dirección: Son todos aquellos de carácter transversal a todo el resto de procesos de la empresa. Relacionados con la estrategia de la empresa.

\section{Cuadro $\mathbf{N}^{\circ} 01$.}

Correlación de las actividades del proceso de internacionalización con los tipos de procesos

\begin{tabular}{|c|c|c|c|}
\hline Correlación & \multicolumn{3}{|c|}{ Actividades del proceso de internacionalización } \\
de las IES \\
\hline $\begin{array}{c}\text { Tipos de Procesos de acuerdo a } \\
\text { Pérez (2009) }\end{array}$ & Estratégicas & Clave & De Apoyo \\
\hline Operativos & & $\mathbf{X}$ & \\
\hline Apoyo & & & $\mathbf{X}$ \\
\hline Gestión & $\mathbf{X}$ & & \\
\hline Dirección & $\mathbf{X}$ & & \\
\hline
\end{tabular}

Cuadro Nº 01. Adaptado de Pérez (2009).

Para los fines de esta investigación se realizó una correlación entre los tipos de procesos propuestos por Pérez (2009) y las actividades del proceso de internacionalización de las IES (Ver cuadro $\mathrm{N}^{\circ}$ 01). El segundo paso es tener de forma gráfica el proceso logístico del sector objeto de estudio, finalmente se añaden en la parte superior las actividades de gestión y dirección (estratégicas), en el centro las actividades operativas (clave) y en la parte inferior las actividades de apoyo.

\section{Metodología.}

Definir el tipo de estudio a realizar es fundamental para todo el trabajo de investigación, ya que según esto se determinan las estrategias, el diseño, los datos que se recolectan, la manera de obtenerlo y otros componentes del diseño. Hernández et al, (2014) define la investigación descriptiva como aquella que implica la descripción, registro, análisis y paráfrasis de la naturaleza 
actual, y la estructura o proceso de los fenómenos enfocándose sobre conclusiones dominantes o sobre cómo una persona, grupo o cosa se conduce o funciona en el presente.

Con relación al objetivo del presente artículo se realizó una investigación descriptiva, bajo un enfoque cualitativo, pues se busca especificar y recoger información sobre las actividades estratégicas, clave y de apoyo del proceso de internacionalización, para su posterior mapeo. En este sentido, la presente investigación muestra un diseño no experimental, transeccional descriptivo debido a que la misma se basa en la búsqueda de información ya existente relacionada a las actividades antes citadas y no pretende modificar o generar nuevos conocimientos.

Finalmente, para la contrastación práctica se utilizó un diseño de investigación relacionado con fuentes de informaciones primarias y secundarias, las cuales fueron personal de las IES entrevistado, así como el análisis de los procesos existentes o documentación relativa a ello que posean las instituciones, respectivamente. Esta búsqueda de información se realizó a través de una investigación de campo, en la cual según Hernández et al (2014), el investigador obtiene los datos del lugar donde se dan los eventos.

Igualmente, se hizo una revisión documental, la cual, de acuerdo a los mismos autores, se hace tomando los hechos recabados y registrados previamente. Los autores que soportaron el desarrollo de la investigación, fueron: Mayer y Catalano (2019), Sorolla (2019)Castro y Wolff (2018), Hoyos y Villamizar (2018), Ganga, Pérez y Manzilla (2018), entre otros.

La población de estudio para el presente trabajo, estuvo representada por todas las Instituciones de Educación Superior Colombianas. Para poder establecer la muestra fue consultada la cantidad de universidades ubicadas en las Ciudades de Medellín y Envigado, por ser los Municipios más poblados del Departamento de Antioquia. En este sentido, la cantidad asciende a 45 instituciones de educación superior. Sin embargo, debido al tiempo que implicó la realización de cada entrevista para el equipo investigador, la muestra objeto de estudio quedó determinada por 4 IESC, todas con certificación de alta calidad y con trayectoria académica y de investigación en el área de internacionalización.

\section{Análisis y Discusión de Resultados.}

Una vez comparada la información teórica de apoyo, con los datos recolectados a través de las entrevistas efectuadas al personal focal de las unidades de Internacionalización de las IES, se procedieron a identificar diferentes actividades, las cuales, considerando su naturaleza, así como su dinamismo e importancia dentro del proceso general, fueron clasificadas como estratégicas, clave y de apoyo, con el propósito de estructurar el mapa del proceso de internacionalización de las IES colombianas (Ver figura $\mathrm{N}^{\circ} 01$ ).

En primer lugar, fueron identificadas tres actividades estratégicas, dentro de las cuales se encuentran: políticas gubernamentales, plan de desarrollo institucional y relaciones de cooperación. Las políticas gubernamentales, tienen como objetivo promover y desarrollar la internacionalización tanto en una ciudad, departamento o país, pues constituyen cambios sustanciales dentro del proceso educativo, lo que implica transformaciones profundas en los modos de pensar y actuar en la educación y es desde ahí donde se debe aplicar este pensamiento global y de internacionalización. La actividad es ejecutada mediante los procesos de políticas regionales, diplomacia educativa y apoyo ministerial a la academia, en pro de fortalecer e incentivar la ejecución del proceso de internacionalización. Según la información recolectada, es pertinente flexibilizar la vinculación del intercambio, modernizando el trámite migratorio, proceso de visado y convalidación de títulos extranjeros. De tal forma que, es necesario una política nacional de internacionalización que ofrezca claridad al gobierno, para priorizar recursos y enmarcar los esfuerzos hacia una estrategia que asegure llevar el proceso hacia otro nivel.

Seguidamente, el plan de desarrollo institucional; éste se puede definir como la visión a futuro, mostrando el direccionamiento institucional y el método que hay que tomar para alcanzarlo. Es considerada una actividad estratégica dentro del proceso de internacionalización ya que puede 
permitir una mayor fluidez, facilitando su ejecución al priorizar recursos en el mismo, además puede mejorar la visibilidad nacional e internacional de la institución, tanto para aceptar estudiantes de otras instituciones, como para generar convenios con otras IES.

Los proyectos de cooperación, como su mismo nombre lo indica, son proyectos cuyo objetivo es generar vínculos con otras IES colombianas, o con grupos de investigadores y profesionales, su naturaleza ya la encamina como una actividad netamente estratégica ya que dichos vínculos pueden generar un mutuo crecimiento de ambas partes.

Es importante destacar, que las IES colombianas están diseñando nuevos métodos de trabajo para ampliar estos convenios no solamente en lo académico y científico, sino también en alianzas que promuevan el desarrollo sostenible de la sociedad en la cual están inmersas.

Continuando con las actividades medulares del proceso de internacionalización, se destaca la movilidad; partiendo de su naturaleza, la movilidad académica de estudiantes, profesores, investigadores y personal administrativo, constituye uno de los fundamentos del proceso de internacionalización. Tal como señala Madarro (2011), en la sociedad del conocimiento de hoy se valoran cada vez más las oportunidades para la realización de estudios y estancias académicas o de investigación en el exterior.

En el caso particular de Colombia, según Castro y Wolf (2018), la Ascun ofrece programas de movilidad académica suscritos con asociaciones de América Latina, en los que además de contar con reconocimiento de estudios, se busca la reciprocidad, como son: el programa maca (con el Consejo Interuniversitario Nacional de Argentina), el programa Macmex (con la Asociación Nacional de Universidades e Instituciones de Educación Superior -Anuies-, de México), y el programa Bracol (con el Grupo Coimbra de Universidades Brasileñas -Gcub-, de Brasil). A partir del 2018, también existe el convenio trilateral Cin-ascun-anuies, denominado Programa pila, así como un programa para facilitar la movilidad de estudiantes de maestría entre la región de Baviera (Alemania) y Colombia, denominado colbay.

En este orden de ideas, de acuerdo a datos del Ministerio de Educación Nacional (2016), al año 2015, había en Colombia 2.293.550 estudiantes matriculados en IES del país, 53.4\% de los cuales estaba inscrito en universidades. De los mismos, tan sólo 11.510 estudiantes habfían realizado algún tipo de movilidad hacia el exterior. No obstante, es importante destacar que a pesar que aproximadamente sólo el $0.5 \%$ de la población inscrita en IES colombianas realiza movilidad internacional, entre el 2011 y el 2015 dicha movilidad creció en promedio un 31\% anual. En cuanto a la movilidad entrante, se observa un crecimiento promedio anual del 55\%, así como un crecimiento en los extranjeros inscritos en programas académicos en el país.

Por otro lado, en relación a la investigación, desde Colciencias, se promueve la internacionalización de la investigación y la innovación colombiana a través del establecimiento y fortalecimiento de relaciones con socios internacionales estratégicos para el desarrollo de la ciencia, la tecnología y la innovación (CTI), así como la gestión de recursos de cooperación internacional y la promoción del conocimiento y practicas innovadoras de Colombia en el extranjero, y viceversa. Lo anterior se gestiona desde los diferentes escritorios geográficos de cooperación con Norteamérica, Asia, África, Oceanía e Iberoamérica (Colciencias, 2017) 
Figura 1. Mapa del Proceso de Internacionalización de las Instituciones de Educación Superior Colombianas

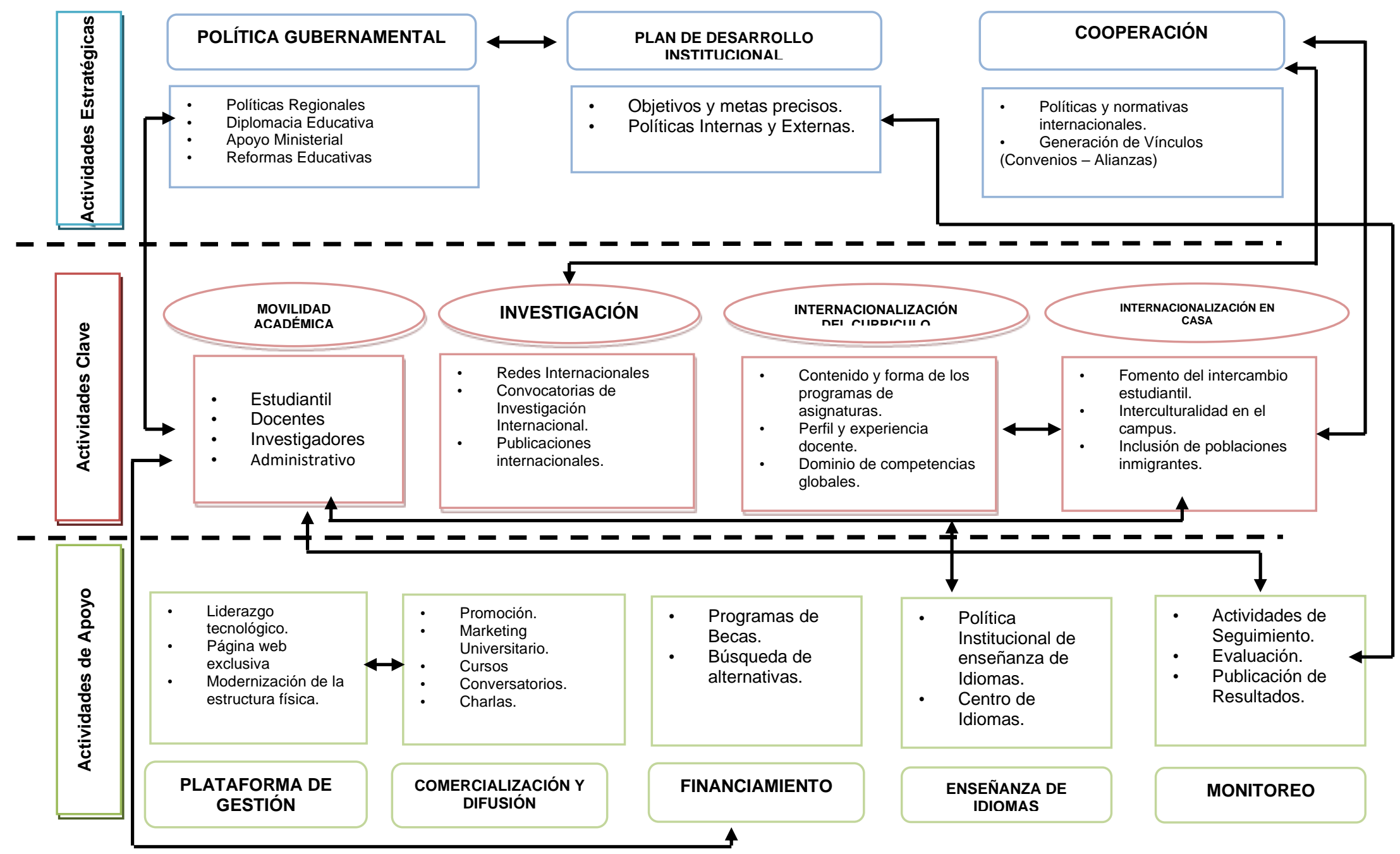

Fuente: elaboración propia. 
Otra actividad clave está constituida por la internacionalización del currículo, la cual puede ser conceptualizada como el conjunto de estrategias y acciones establecidas en el proyecto institucional, orientadas a diseñar un currículo cuyo contenido y forma contribuya a formar estudiantes, propios y extranjeros, para desempeñarse con éxito, profesional y socialmente, en contextos internacionales y multiculturales. A este respecto, Madera (2005), opina que un currículum internacionalizado se fundamenta en la integración de la perspectiva internacional en la función de docencia, lo que incluye: contenidos y diseño de los programas de curso, métodos didácticos, sistemas de evaluación, investigación y extensión. El propósito de incluir esta concepción en la cultura de las instituciones educativas, es formar ciudadanos capaces de actuar social y profesionalmente en un contexto internacional, interdisciplinario y multicultural.

Desde este punto de vista, la internacionalización del currículo se percibe como la incorporación de la concepción internacional en todos los elementos de la propuesta curricular y no solamente en el planeamiento de los cursos, así mismo, sus beneficios no deben esperarse únicamente en lo económico, sino también vistos como una oportunidad de crecimiento sociocultural de los individuos y las instituciones. En este sentido, Camillioni (2010), opina que se trata de una tendencia consolidada, que conlleva a desarrollar experiencias interculturales, de diferentes maneras, para aprender a convivir con otros. De esta forma, se visualiza la internacionalización del currículum como la unión de contenidos y metodologías de enseñanza que le faciliten a los participantes, indagar la dimensión internacional dentro de su disciplina, comprender y respetar la diversidad nacional y la de otros países, adquiriendo competencias comunicativas que le aseguren responder de manera asertiva a nuevas formas de interrelación.

Siguiendo con el orden de ideas, es importante resaltar algunos resultados mostrados por Castro y Wolff (2018) acerca de la Encuesta regional sobre tendencias de la internacionalización en educación terciaria en América Latina y el Caribe, realizada por el Observatorio Regional sobre Internacionalización y Redes en Educación Terciaria (OBIRET) del Instituto Internacional para la Educación Superior en América Latina y el Caribe de la Organización de las Naciones Unidas para la Educación, la Ciencia y la Cultura (Unesco-Iesalc).

En cuanto a la internacionalización del currículo, la encuesta señala que la mayoría (51\%) de las Instituciones de Educación Superior Terciarias (IET) indican no contar con una política de internacionalización del currículo; dentro de las actividades de internacionalización del currículo, la que se destaca por la frecuencia con la que se realiza, es la movilidad saliente de estudiantes (87\%), seguida por la movilidad entrante de estudiantes (75\%) y la invitación de profesores extranjeros para realizar actividades académicas en la institución (73\%); el 72\% indica no ofrecer cursos masivos abiertos online (Mooc, por sus siglas en inglés), y el $82 \%$ indica no brindar la modalidad de movilidad virtual. A este respecto, las barreras evidenciadas para incrementar la internacionalización del currículo, recaen en problemas administrativos, además de aquellas alusivas con la transferencia de créditos, las divergencias de calendarios escolares, normas institucionales rígidas y la ausencia de política institucional (Castro y Wolff, 2018).

A partir de los datos antes citados, es oportuno duplicar esfuerzos en formalizar programas académicos internacionalizados para aquellos participantes que no tienen la oportunidad de efectuar una estancia internacional, a través de programas innovadores como los académicos colaborativos con IET internacionales (doble titulación y movilidad virtual), y estimular el dominio de idiomas extranjeros en toda la comunidad académica. La internacionalización de la investigación necesita ser promovida de una forma más metódica y con mayores recursos para que Colombia alcance un mayor nivel de producción de conocimiento (Hoyos y Villamizar, 2018).

Ahora bien, en el caso colombiano, vale destacar algunos programas ejecutados por el Instituto Colombiano de Crédito Educativo y Estudios en el Exterior Mariano Ospina Pérez (ICETEX), en cuanto a la promoción de la internacionalización del currículo y de la investigación. En primer lugar, el programa Asistentes de Idiomas, el cual apoya la movilidad académica de docentes de idiomas extranjeros para incentivar y contribuir en el proceso de fortalecimiento de los 
departamentos de idiomas de colegios e IES. Entre el 2011 y el 2016, se han movilizado más de setecientos asistentes de idiomas en el país en el marco de dicho programa. El segundo es el Programa de Profesores Invitados, recientemente titulado Fellows Colombia, promueve la movilidad de expertos internacionales hacia IES y centros de investigación colombianos con el objetivo de fortalecer programas académicos y de investigación, las estancias pueden tener una duración de hasta seis meses de acuerdo con los rubros financiados por el programa, y en el marco del mismo se han invitado 4.600 expertos, 30\% de los cuales estuvo en Colombia en el 2016. (Instituto Colombiano de Crédito Educativo y Estudios en el Exterior Mariano Ospina Pérez, 2017).

Para todas las IES colombianas entrevistadas, existe una actividad que ha cobrado vigencia en los últimos días y es la internacionalización en casa, siendo un medio efectivo que permite a los estudiantes la tradicional experiencia de vivir la internacionalización, desde actividades desarrolladas "en casa", reforzando valores de interculturalidad, diversidad e inclusión. De esta manera, IES jóvenes, que no disponen de una fuerte red internacional, a través de estas experiencias de trabajo en red, han logrado incorporar buenas prácticas de universidades nacionales e internacionales con mayor tradición. En este contexto, vale destacar el programa implementado por la Universidad de Antioquia, denominado Parceros, creado como una iniciativa de acompañamiento voluntario por parte de los propios estudiantes; dentro de las funciones básicas, están: información de los lugares de esparcimiento, realizar charlas, conversatorios, compartir costumbres, hábitos culturales, entre otros.

Bajo este mismo orden de ideas, también fueron identificadas unas actividades que soportan la ejecución efectiva del proceso de internacionalización, tal como son las actividades de apoyo. En este sentido, para Gacel Ávila (2009) el nivel de compromiso por parte de las autoridades académicas con el proceso de internacionalización, puede observarse en la importancia que se le concede en la estructura organizativa a la perspectiva internacional, además de los recursos humanos y el financiamiento que se otorga para dicho proceso.

A partir de este contexto, las plataformas de gestión universitaria, deben facilitar la información necesaria para orientar a estudiantes interesados en la internacionalización, estableciendo un campus digital y un campus físico enfocado en el intercambio académico; apoyándose en la tecnología como herramienta estratégica para la modernización y mejoramiento de la estructura física y del personal. Igualmente, para respaldar el proceso de comercialización y difusión, deben contar con una plataforma web exclusiva para el asesoramiento y publicidad que permita mayor acogida en el ámbito estudiantil, además de realizar charlas, conversatorios, entre otras actividades divulgativas.

Ahora bien, de acuerdo a las entrevistas realizadas, para elevar la participación de los estudiantes interesados en un intercambio académico, las universidades deben promoverlo a través de diferentes programas y acuerdos que permitan mayor bienestar estudiantil, es decir, implementar planes de financiamiento, según Abba (2015), en cuanto a universidades que reúnen una cantidad considerable de recursos financieros como de matrícula docentes, estudiantes e investigadores, presentan un mayor desarrollo de la planificación estratégica, gestión de programas y proyectos, decisión política de las autoridades universitarias y capacitación de los recursos humanos". Este apoyo financiero refleja grandes oportunidades para todos los estudiantes y desarrolla programas como becas internacionales, que le permitan a los estudiantes centrar todos sus esfuerzos en su formación académica, enalteciendo así la calidad de su educación y la imagen internacional de la universidad. También permite mejorar y ampliar la búsqueda de alternativas de movilidad estudiantil, sean acuerdos con otras instituciones universitarias en pro del estudiante interesado, acuerdos con empresa, multinacionales y, sobre todo, con el gobierno para favorecer extensamente el desarrollo nacional.

Otra de las funciones básicas de apoyo al proceso de internacionalización, es la enseñanza de idiomas; lo que hace necesario que las IES colombianas dispongan de un centro de idiomas, o la incorporación de lenguas en el pensum académico, ya que es de gran importancia para aquellas 
universidades que buscan una expansión, o un simple ajuste, según la demanda laboral actual, por medio de la internacionalización, para el alumnado, docentes e investigadores.

Finalmente, se identificó el proceso de monitoreo, que consiste en llevar un seguimiento y evaluación de los resultados, definido como apoyo, ya que concibe una supervisión de todos los otros procesos y así mismo, una evaluación efectiva general de la internacionalización. En otras palabras, es una actividad transversal en todas las demás actividades mencionadas anteriormente, ya que se debe establecer una comparación entre lo definido en el plan de desarrollo institucional y los objetivos alcanzados en cada periodo académico. Igualmente, implica un correcto seguimiento hacia los estudiantes en sus avances y su incorporación cultural, publicación de resultados al terminar el proceso, y finalmente una evaluación continua de todo el proceso de internacionalización con la finalidad de identificar desviaciones y establecer acciones para mejorar. En síntesis, el alcance de este trabajo es un primer acercamiento a todas aquellas actividades que conforman el ejercicio de la internacionalización de las instituciones de educación superior colombianas. Vale resaltar que, aunque existen gran cantidad de documentos - los cuales han sido abordados en esta investigación -, todos están orientados en describir qué hacen las instituciones, el Gobierno y otros gremios en pro de fomentar dicha actividad; no obstante, ninguno aplica la gestión por procesos a fin generar una estructura de manera gráfica para cada actividad, facilitando la dirección de los esfuerzos en aquellas que realmente agregan valor al proceso.

\section{Conclusiones}

Una vez culminada la investigación, se logró el objetivo principal de la misma, al estructurar el mapa del proceso de internacionalización de las Instituciones de Educación Superior Colombianas (IESC). La ejecución de las entrevistas efectuadas a los líderes de departamento de internacionalización, ofreció información que coadyuvó en la clasificación de todas las actividades del proceso, ubicándolas en tres áreas: estratégicas, clave y de apoyo.

En este sentido, a nivel estratégico se concluye que las políticas gubernamentales, el plan de desarrollo institucional y las relaciones de cooperación, sirven para encaminar la ejecución del proceso de internacionalización. Sin embargo, existe la necesidad de disponer de una política nacional de internacionalización, con el propósito de tener claridad en la priorización de recursos, además de enmarcar los esfuerzos en estrategias que permitan llevar la educación superior colombiana a otro nivel.

Con respecto a las actividades clave, quedaron determinadas por la movilidad académica, la investigación, internacionalización del currículo y la internacionalización en casa, ésta última, siendo una de las funciones más novedosas incorporadas por las IESC, facilitando la posibilidad de tener la experiencia de vivir la internacionalización, desde actividades desarrolladas "en casa", reforzando valores de interculturalidad, diversidad e inclusión. En términos generales, las actividades medulares del proceso, ha tenido un impulso significativo promovido por acuerdos internacionales que han mejorado la visibilidad de Colombia ante el mundo, haciéndola más atractiva para intercambios académicos y de investigación.

Finalmente, las actividades de soporte o apoyo al proceso, están constituidas por la plataforma de gestión universitaria, la comercialización y difusión, el financiamiento y monitoreo a todo el sistema. En este contexto, las IESC vienen desarrollando funciones que respaldan el desarrollo efectivo de las actividades antes mencionadas, a pesar que, es vital esfuerzos mancomunados entre el gobierno y la academia, para respaldar financieramente la promoción y alcance de los proyectos de internacionalización.

El mapeo realizado requiere de investigaciones más detalladas, con el propósito de construir un modelo de gestión, capaz de medir y monitorear continuamente el nivel de efectividad en el desarrollo de cada actividad. Así mismo, es vital un trabajo coordinado entre el Gobierno colombiano y las IESC, a fin de proponer acciones contundentes y no quedarse únicamente en planes y políticas de gestión. De tal forma que, las IES no pueden actuar aisladas, requieren un 
acople preciso entre el gobierno y el sector privado en general, integrando dicho relacionamiento en las funciones sustantivas de la universidad.

\section{Referencias Bibliográficas}

Abba, María. (2015). Las oficinas de relaciones internacionales en el proceso de internacionalización de la educación superior. Un análisis a través de variables de gestión. Revista de Gestión Universitaria en Ámerica Latina - GUAL. Vol. 8, № 04. Brasil (Pp 20-37).

Bello, Carlos. (2006). Manual de Producción. (Segunda edición). Editorial Ecoe. Colombia.

Benavides, Carlos y Quintana Cristina. (2003). Gestión del conocimiento y calidad total. Ediciones Díaz de Santos. México.

Botero Montoya, Luis., y Bolívar García, María. (2015). Guías para la internacionalización de la educación superior. Gestión de la internacionalización. Ministerio de Educación Nacional. Bogotá.

Camillioni, Alicia. (2010). Gestión curricular en las instituciones de Educación Superior. Conferencia de Red Interinstitucional de Formación Docente, RIFED. 2-9-2010. Universidad de Costa Rica. Costa Rica.

Castro, Alma y Wolf, Marcela. (2018). Internacionalización de la Educación Superior en América Latina y el Caribe. Caso Colombia. Red Regional para el Fomento de la Internacionalización de la Educación Superior en América Latina (RIESAL). Colombia.

Colciencias (2017). Internacionalización. Recuperado de https://www.colciencias.gov.co/portafolio/internacionalizacion. Consulta: 15/04/2019.

Colotta, Mariana; Lavallen, Fabian y Presa, Adriana. (2017). Internacionalización: perspectivas y debates en la universidad Latinoamericana. Boletín Científico. Vol. 7, No 01. Argentina. (Pp 98-111).

Gacel Ávila, Jocelyn. (2009). Modelo de Oficinas de Relaciones Internacionales, en: Casos Prácticos para la Gestión de la Internacionalización en la Universidades. Red Safiro II. España.

Ganga, Francisco; Pérez, Armenio y Mansilla, Juan. (2018). Paradigmas emergentes en la gobernanza universitaria: una aproximación teórica. Revista internacional de filosofía y teoría social. Año 23, No 83. Venezuela (Pp 123-136).

Gómez, Ignacio. (2009). Enfoque a procesos. Principios ISO 9001. Extraído de: https://hederaconsultores.blogspot.com/2009/06/enfoque-procesos-principios-iso9001.html. Consulta: 22/04/2019.

Harrington, James. (1993). Mejoramiento de los procesos de la empresa. (Primera edición). Editorial Mc Graw Hill. Colombia.

Hernández, Roberto; Fernández, Carlos; Baptista, Pilar. (2014) Metodología de la investigación. (Sexta edición). Editorial Mc Graw Hill. México.

Hoyos, Juan y Villamizar, Luisa. (2018). Asociaciones latinoamericanas que impulsan la internacionalización de la educación superior. Caso Colombia. Red Regional para el Fomento de la Internacionalización de la Educación Superior en América Latina (RIESAL). Colombia.

Instituto Colombiano de Crédito Educativo y Estudios en el Exterior Mariano Ospina Pérez (2017). Colombia Científica. Recuperado de http://colombiacientifica.gov.co/colombia/componentes/pasaporte-a-la-ciencia/.

Consulta: 24/04/2019.

Madarro, Ana. (2011). Redes de Movilidad Académica para la Cooperación. Revista Iberoamericana de Educación. $N^{\circ}$ 57. España (Pp 71-107).

Madera, Inmaculada. (2005). Un nuevo paradigma educativo: la internacionalización del currículum en la era global. Universidad APEC. Ponencia en el Cuarto encuentro de 
Educación y Pensamiento, 6 al 8 de octubre del 2005. República Dominicana. Extraído de http://ofdp rd.tripod.com/encuentro4/apec.html. Consulta: 13/04/2019.

Mayer, Liliana y Catalano, Bárbara. (2019). Internacionalización de la educación y movilidad: reflexiones a partir del caso argentino. Revista de Ciencias Sociales y Humanas. N 29. Ecuador (Pp 19-41).

Ministerio de Educación Nacional (2016). Ministerio de Educación Nacional de Colombia. Extraído de http://www.mineducacion.gov. Consulta: 15/04/2019.

Pérez, Jorge. (2009). Gestión por Procesos. (Tercera Edición). Editorial ESIC, España.

Sorolla, Ileana. (2019). La internacionalización ¿’oportunidad, meta o quimera para una institución de educación superior? Revista Dilemas Contemporáneos: educación, política y valores. Año 6, $\mathrm{N}^{\circ}$ 02. México (Pp 1-30) 